\title{
Cell culture of human gingival fibroblasts, oral cancer cells and mesothelioma cells with serum-free media, STK1 and STK2
}

\author{
YUTA TSUGENO $^{1}$, FUYUKI SATO ${ }^{1,2}$, YASUTERU MURAGAKI ${ }^{2}$ and YUKIO KATO ${ }^{3}$ \\ ${ }^{1}$ Department of Pathology and Bioscience, Hirosaki University Graduate School of Medicine, Hirosaki, Aomori 036-8562; \\ ${ }^{2}$ First Department of Pathology, Wakayama Medical University School of Medicine, Wakayama 641-0012; \\ ${ }^{3}$ Department of Dental and Medical Biochemistry, Hiroshima University Graduate School of Biomedical Science, \\ Hiroshima 734-8553, Japan
}

Received May 13, 2014; Accepted June 25, 2014

DOI: 10.3892/br.2014.306

\begin{abstract}
The majority of cells are cultured with Dulbecco's modified Eagle's medium (DMEM) or RPMI supplemented with fetal bovine serum (FBS), which contains numerous factors, including cytokines, nutrients and unknown growth factors. These factors may affect cell growth, apoptosis and differentiation. The serum-free medium, STK2, has been previously reported as suitable for the cell culture of human mesenchymal stem cells. However, how STK1 or STK2 affect the cell proliferation of normal and cancer cells remains unknown. The present study examined the growth of the human gingival fibroblast (HGF-1) cell-line and the HSC-3, CA9-22 and MSTO cancer cell-lines, cultured with STK1 and STK2. STK1 increased the cell proliferation of HGF-1 compared to DMEM by assessment with the 3-(4,5-dimethylthiazol-2-yl)-5-(3-carboxymethoxyphenyl)-2-(4-sulfophenyl)2H-tetrazolium (MTS) assay, whereas STK1 and STK2 markedly inhibited the cell proliferation of HSC-3 and MSTO. The cell proliferation rate of CA9-22 cultured with STK1 or STK2 for $96 \mathrm{~h}$ was $\sim 2$-fold higher than the rate for $24 \mathrm{~h}$ culture. The shape of the HSC-3 cells was also found to have changed to round when cultured with STK2. These results indicate that STK1 increased the cell proliferation of HGF-1 compared to DMEM, whereas the proliferation of HSC-3 and MSTO was inhibited by STK1 and STK2. Thus, STK1 and STK2 had different affects on the cell growth of HGF-1, CA9-22, HSC-3 and MSTO.
\end{abstract}

Correspondence to: Dr Fuyuki Sato, First Department of Pathology, Wakayama Medical University School of Medicine, 811-1 Kimiidera, Wakayama 641-0012, Japan

E-mail: fsatoDEC1DEC2@yahoo.co.jp

Key words: STK1, STK2, fetal bovine serum, fetal calf serum, MTS assay

\section{Introduction}

Fetal bovine or calf serum (FBS or FCS, respectively) includes unspecified amounts of growth factors, which may modify the immune response and cell differentiation (1-4). In addition, cell growth and differentiation may be affected in cell culture, depending on the amount of FBS or FCS present.

A previous study demonstrated that FBS inhibited cell growth and increased apoptosis (4). Compared to FBS-supplemented media, serum-free media does not differ between individual batches, and thus, it may be suitable for the cell culture performance. There are several types of serum-free media, specific media is suitable for stem cells and others are acceptable for primary cell culture $(5,6)$. These serum-free media do not affect cell growth and differentiation in cell culture. STK2 is a serum-free medium that has been developed for use with human mesenchymal stem cells (hMSC) (7). STK2 had a minimal effect on the gene expression and morphology of hMSC after 50 days of culture, whereas Dulbecco's modified Eagle's medium (DMEM) with 10\% FBS significantly changed the expression of $\sim 1,000$ genes and the cell morphology (7). Although STK2 is suitable for hMSC culture, it remains unknown whether STK2 can promote the growth of normal and cancer cells.

CA9-22 and HSC-3 are human oral squamous cell carcinoma cell lines that are often used for in vitro assays. CA9-22 highly expresses epidermal growth factor receptor (EGFR), whereas HSC-3 is prone to lymph node metastasis $(8,9)$. Compared to HSC-3, it appears likely that histone deacetylase 2 is highly expressed in CA9-22 (10). MSTO is a human malignant mesothelioma cell-line that highly expresses EGFR (11). These cells have been cultured previously using general medium with $10 \%$ FBS (12-14). The present study aimed to examine how STK1 or STK2 affected the cell growth of human gingival fibroblasts (HGF-1), CA9-22, HSC-3 and MSTO by the 3-(4,5-dimethylthiazol-2-yl)-5-(3-carboxymethoxyphenyl)-2-(4-sulfophenyl)-2H-tetrazolium (MTS) assay. STK1 increased the proliferation of HGF-1 compared to DMEM, whereas STK1 and STK2 inhibited the proliferation of HSC-3 and MSTO. CA9-22 was able to grow with STK, but the proliferation rate of CA9-22 cultured with STK was lower than with DMEM. These results indicate that STK affects cell 
$\mathbf{A}$

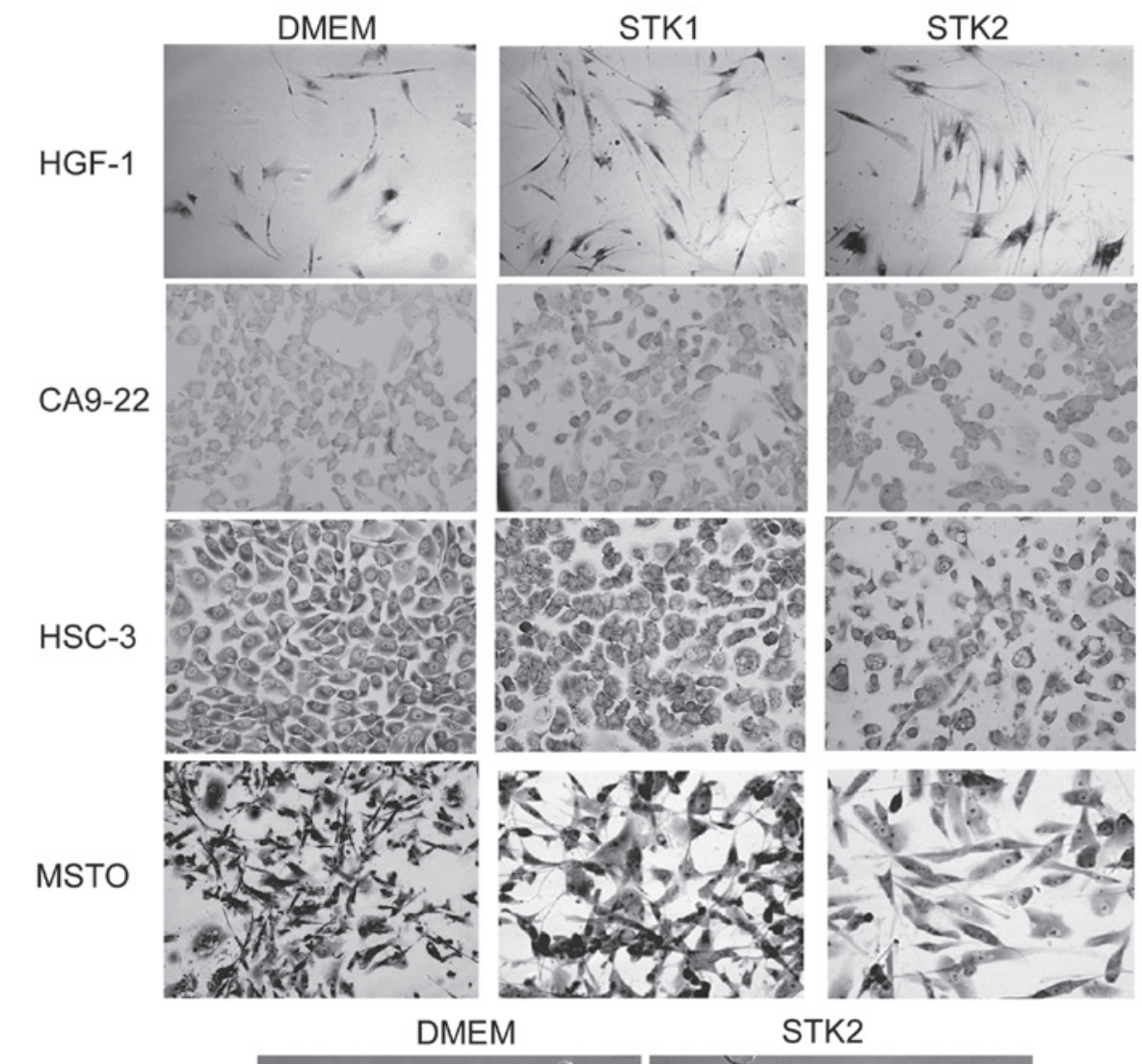

B

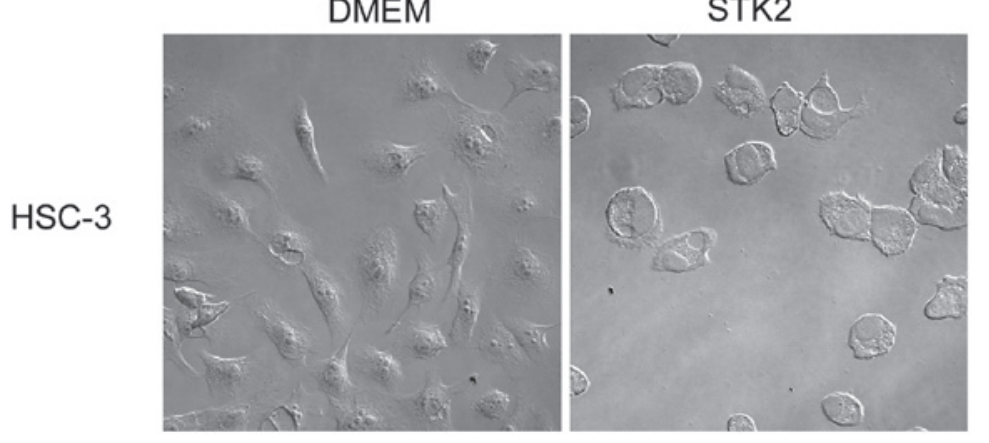

Figure 1. Dulbecco's modified Eagle's medium (DMEM), STK1 and STK2 show differential effects on cell proliferation. (A) Human gingival fibroblast (HGF-1), CA9-22, HSC-3 or MSTO cells were cultured in chamber slides. The culture medium was changed to DMEM, STK1, or STK2 after $24 \mathrm{~h}$ and the cells were cultured for an additional $96 \mathrm{~h}$ before fixation. (B) HSC-3 cells were cultured with DMEM or STK2 and were fixed. Images were captured in bright-field mode.

growth in the different cell lines, HGF-1, CA9-22, HSC-3 and MSTO.

\section{Materials and methods}

Cell culture. HGF-1 and MSTO mesothelioma cells were obtained from the American Type Culture Collection (Manassas, VA, USA). CA9-22 and HSC-3 human oral cancer cell lines were obtained from the Japanese Cancer Research Resources Bank (Tokyo, Japan). These cells were cultured with DMEM-high glucose (Sigma-Aldrich, St Louis, MO, USA) supplemented with 10\% FBS, STK1 or STK 2 serum-free media (DS Pharma Biomedical Co., Ltd., Osaka, Japan) at $37^{\circ} \mathrm{C}$ in a humidified atmosphere of $95 \%$ air and $5 \% \mathrm{CO}_{2}$. Images of the cells were captured using a Zeiss microscope (Carl Zeiss, Oberkochen, Germany).

Cell viability assay. The cell viability assay was performed using the MTS assay. The cells were seeded in 96-well plates.
After culturing for $24 \mathrm{~h}$, the culture medium was changed to DMEM, STK1 or STK2, and the culture was continued for 96 h. CellTiter $96^{\circledR} \mathrm{AQ}_{\text {ueous }}$ One Solution reagent (Promega Corporation, Madison, WI, USA) was added to each well and the cells were incubated at $37^{\circ} \mathrm{C}$ for $1 \mathrm{~h}$. The absorbance $\left(\mathrm{OD}_{490} \mathrm{~nm}\right)$ was measured using a 96-well plate reader of iMark Microplate Absorbance Reader (Bio-Rad, Tokyo, Japan).

Statistical analysis. The data are provided as mean \pm standard deviation. Student's t-test was used for statistical analysis $(\mathrm{P}<0.05)$.

\section{Results}

STK inhibits the cell proliferation of HSC-3 and MSTO. The affect of STK1 or STK2 was examined with regards to the cell growth of normal and cancer cells by the MTS assay. HGF-1, CA9-22, HSC-3 and MSTO cells were cultured with DMEM, STK1, or STK2 for 24-96 h. The cell proliferation of 

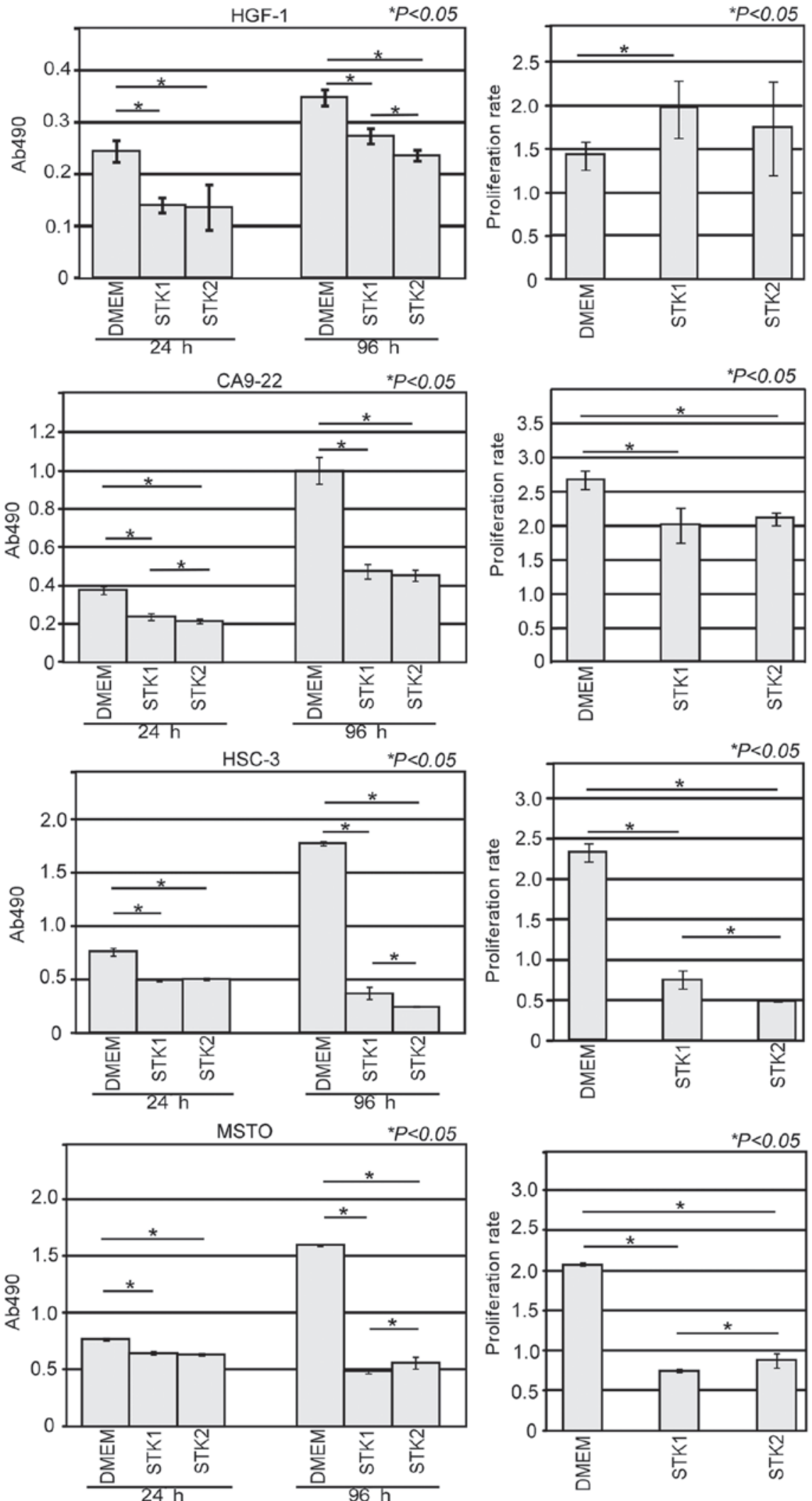

Figure 2. Quantification of human gingival fibroblast (HGF-1), CA9-22, HSC-3 and MSTO cell proliferation at 24 and 96 h when cultured with Dulbecco's modified Eagle's medium (DMEM), STK1 and STK2. The cells were cultured and the MTS-assay was performed. The left panel shows the raw cell proliferation data at 24 and $96 \mathrm{~h}$ of cell culture; the right panel shows the cell proliferation rate as determined by Ab490 $\mathrm{nm}$ at $96 \mathrm{~h}$, divided by the value at $24 \mathrm{~h}$.

HSC-3 at $96 \mathrm{~h}$ after the medium was changed was markedly inhibited by STK1 and STK2. For MSTO, some floating cells were observed when cultured with STK1 (Fig. 1A). STK2 also affected the cell morphology of HSC-3, which changed to a round shape (Fig. 1B). The cell proliferation rates of HGF-1 cultured with DMEM, STK1 and STK2 were 1.4, 2.0 
and 1.7-fold, respectively (Fig. 2). There was a significant increase in the cell proliferation rate between DMEM and STK1 $(\mathrm{P}<0.05)$, whereas there was no significant difference between DMEM and STK2 or STK1 and STK2. The cell proliferation rates of CA9-22 cultured with DMEM, STK1 and STK2 were 2.7, 2.0 and 2.1-fold, respectively. There was a significant decrease in the cell proliferation rate between DMEM and STK1, or DMEM and STK2 $(\mathrm{P}<0.05)$, whereas there was no significant difference in the cell proliferation rate between STK1 and STK2. The cell proliferation rates of HSC-3 cultured with DMEM, STK1 and STK2 were 2.3, 0.8 and 0.5 -fold, respectively. There were significant decreases in the cell proliferation rates between DMEM and STK1, between DMEM and STK2 $(\mathrm{P}<0.05)$, and between STK1 and STK2 $(\mathrm{P}<0.05)$. The cell proliferation rates of MSTO cultured with DMEM, STK1 and STK2 were 2.1, 0.7 and 0.9 -fold, respectively. There were significant decreases in the cell proliferation rates between DMEM and STK1, and DMEM and STK2 $(\mathrm{P}<0.05)$, and there was also a significant increase in the cell proliferation rate between STK1 and STK2 $(\mathrm{P}<0.05)$.

\section{Discussion}

Previously, certain studies have performed cell culture without FBS or FCS for pre-clinical data or embryonic stem cells, as the serum affects the cell culture performance (15-17). STK1 and STK2 are serum-free media with unclear roles in the culture of normal and cancer cells. In the present study, the STK media were demonstrated to inhibit the cell proliferation of HSC-3 and MSTO, whereas they increased the cell proliferation of HGF-1 and CA9-22. When cultured with DMEM, these cancer cells grew well compared to STK1 and STK2. There are several possible explanations as to why STK media inhibited HSC-3 and MSTO cell proliferation. First, STK may have induced apoptosis or cell cycle arrest in HSC-3 and MSTO cells, as STK2 was found to induce a round shape in HSC-3. In addition, MTSO cultured with STK1 gave rise to a number of floating or detached cells. Whether this was due to apoptosis or detachment, and the explanation for why STK1 did not induce as many floating cells with HSC-3, could not be elucidated. STK1 may also possibly affect MSTO cell adhesion molecules, including $\beta$-catenin, claudins and E-cadherin $(18,19)$.

CA9-22 and MSTO highly express EGFR, which may give rise to different proliferation rates between these two cell types when cultured with STK. Additionally, other factors may regulate cell proliferation, including the clock genes, PERI and $P E R 3$, which are closely associated with cisplatin-induced apoptosis in CA9-22 (8,20). PER1 is highly expressed in CA9-22, whereas it is weakly expressed in HGF-1. Deleted in esophageal cancer 1 (DEC1) regulates cell proliferation under a serum-free condition (21). These molecules are closely associated with the regulation of apoptosis, cell cycle arrest and cell proliferation $(20,22,23)$. Therefore, it can be speculated that PER1, PER3 and DEC1 may be involved in the regulation of cell proliferation by STK. These studies indicate that HSC-3 and MSTO cells may require more nutritional components than HGF-1 and CA9-22. Therefore, future studies should clarify how STK affects apoptosis and the cell cycle.

\section{Acknowledgements}

The present study was supported in part by Grants-in-Aid for Science from the Ministry of Education, Culture, Sports, Science and Technology of Japan (to Y.M. and Y.K.).

\section{References}

1. Kading VH, Blalock JE and Gifford GE: Effect of serum on the antiviral and anticellular activities of mouse interferon. Arch Virol 56: 237-242, 1978.

2. Toldbod HE, Agger R, Bolund L and Hokland M: Potent influence of bovine serum proteins in experimental dendritic cell-based vaccination protocols. Scand J Immunol 58: 43-50, 2003.

3. Qin ZL, Ju HP, Liu Y, Gao TT, Wang WB, Aurelian L, Zhao P and Qi ZT: Fetal bovine serum inhibits hepatitis $C$ virus attachment to host cells. J Virol Methods 193: 261-269, 2013.

4. Roberg K, Ceder R, Farnebo L, Norberg-Spaak L and Grafström RC: Multiple genotypic aberrances associate to terminal differentiation-deficiency of an oral squamous cell carcinoma in serum-free culture. Differentiation 76: 868-880, 2008.

5. Liu ZZ, Chen P, Lu ZD, Cui SD and Dong ZM: Enrichment of breast cancer stem cells using a keratinocyte serum-free medium. Chin Med J (Engl) 124: 2934-2936, 2011.

6. Mark P, Kleinsorge M, Gaebel R, Lux CA, Toelk A, Pittermann E, David R, Steinhoff G and Ma N: Human mesenchymal stem cells display reduced expression of CD105 after culture in serum-free medium. Stem Cells Int 2013: 698076, 2013.

7. Sawada R, Yamada T, Tsuchiya T and Matsuoka A: Microarray analysis of the effects of serum-free medium on gene expression changes in human mesenchymal stem cells during the in vitro culture. Yakugaku Zasshi 130: 1387-1393, 2010 (In Japanese).

8. Hirai M, Gamou S, Minoshima S and Shimizu N: Two independent mechanisms for escaping epidermal growth factor-mediated growth inhibition in epidermal growth factor receptor-hyperproducing human tumor cells. J Cell Biol 107: 791-799, 1988.

9. Matsui T, Ota T, Ueda Y, Tanino M and Odashima S: Isolation of a highly metastatic cell line to lymph node in human oral squamous cell carcinoma by orthotopic implantation in nude mice. Oral Oncol 34: 253-256, 1998.

10. Chang CC, Lin BR, Chen ST, Hsieh TH, Li YJ and Kuo MY: HDAC2 promotes cell migration/invasion abilities through HIF-1 $\alpha$ stabilization in human oral squamous cell carcinoma. J Oral Pathol Med 40: 567-575, 2011.

11. Jänne PA, Taffaro ML, Salgia R and Johnson BE: Inhibition of epidermal growth factor receptor signaling in malignant pleural mesothelioma. Cancer Res 62: 5242-5247, 2002.

12. Bourguignon LY, Earle C, Wong G, Spevak CC and Krueger K: Stem cell marker (Nanog) and Stat-3 signaling promote MicroRNA-21 expression and chemoresistance in hyaluronan/CD44-activated head and neck squamous cell carcinoma cells. Oncogene 31: 149-160, 2012.

13. Yamamoto K, Tominaga K, Sukedai M, Okinaga T, Iwanaga K, Nishihara $\mathrm{T}$ and Fukuda J: Delivery of cytolethal distending toxin B induces cell cycle arrest and apoptosis in gingival squamous cell carcinoma in vitro. Eur J Oral Sci 112: 445-451, 2004.

14. Chiang SL, Jiang SS, Wang YJ, Chiang HC, Chen PH, Tu HP, Ho KY, Tsai YS, Chang IS and Ko YC: Characterization of arecoline-induced effects on cytotoxicity in normal human gingival fibroblasts by global gene expression profiling. Toxicol Sci 100: 66-74, 2007.

15. Kim SJ and Diamond B: Generation and maturation of bone marrow-derived DCs under serum-free conditions. J Immunol Methods 323: 101-108, 2007.

16. Li Y, Yokohama-Tamaki $\mathrm{T}$ and Tanaka TS: Short-term serum-free culture reveals that inhibition of Gsk3 $\beta$ induces the tumor-like growth of mouse embryonic stem cells. PLoS One 6: e21355, 2011.

17. Millington M, Arndt A, Boyd M, Applegate T and Shen S: Towards a clinically relevant lentiviral transduction protocol for primary human CD34 hematopoietic stem/progenitor cells. PLoS One 4: e6461, 2009. 
18. Fassina A, Cappellesso R, Guzzardo V, Dalla Via L, Piccolo S, Ventura L and Fassan M: Epithelial-mesenchymal transition in malignant mesothelioma. Mod Pathol 25: 86-99, 2012

19. Ikari A, Atomi K, Takiguchi A, Yamazaki Y, Hayashi H, Hirakawa J and Sugatani J: Enhancement of cell-cell contact by claudin-4 in renal epithelial Madin-Darby canine kidney cells. J Cell Biochem 113: 499-507, 2012.

20. Sato F, Wu Y, Bhawal UK, Liu Y, Imaizumi T, Morohashi S, Kato Y and Kijima H: PERIOD1 (PER1) has anti-apoptotic effects, and PER3 has pro-apoptotic effects during cisplatin (CDDP) treatment in human gingival cancer CA9-22 cells. Eur J Cancer 47: 1747-1758, 2011.

21. Li Y, Zhang H, Xie M, Hu M, Ge S, Yang D, Wan Y and Yan B: Abundant expression of Dec1/stra13/sharp2 in colon carcinoma: its antagonizing role in serum deprivation-induced apoptosis and selective inhibition of procaspase activation. Biochem J 367: 413-422, 2002.
22. Bhawal UK, Sato F, Arakawa Y, Fujimoto K, Kawamoto T, Tanimoto K, Ito Y, Sasahira T, Sakurai T, Kobayashi M, et al: Basic helix-loop-helix transcription factor DEC1 negatively regulates cyclin D1. J Pathol 224: 420-429, 2011.

23. Gery S, Komatsu N, Baldjyan L, Yu A, Koo D and Koeffler HP: The circadian gene perl plays an important role in cell growth and DNA damage control in human cancer cells. Mol Cell 22: 375-382, 2006 